\title{
Diseño de un modelo de gestión para el desarrollo de las pequeñas unidades agrícolas rurales del Perú: Talleres de asociatividad
}

\section{RESUMEN}

Uno de los principales problemas que enfrentan las pequeñas unidades agricolas rurales es establecer e implementar un modelo de gestión que aproveche sus potencialidades e impulse su desarrollo productivo. Con miras a suplir dicha carencia, se realizó una investigación de tipo transversal, exploratoria-descriptiva, no experimental y prospectiva entre los años 2008 y 2011. Su finalidad principal fue generar un modelo de desarrollo para una entidad autónoma e integrar tres sectores (público, privado y académico) de tal manera que garantizaran el aprovechamiento de los recursos de manera sostenible y competitiva, a partir del desarrollo de la vocación agropecuaria, el recurso hídrico, el parque tecnológico, las actividades no tradicionales (turismo y acuicultura) y la gestión pública.

Palabras claves: virú, asociatividad, consorcios de exportación, teoría de bienes comunes.

DESIGN OF A MODEL OF MANAGEMENT FOR THE DEVELOPMENT OF THE SMALL AGRICULTURAL RURAL UNITS OF PERU ASOCIATIVIDAD'S WORKSHOPS

\section{ABSTRACT}

One of the main problems faced by small rural farming units is to develop and implement managerial models that contribute to leverage their natural potential and boost their productive development. In order to mitigate the impact of this problem, a cross-section, exploratorydescriptive, non-experimental and prospective research was carried on, between 2008 and 2011. The main propose of this research was to design a model for autonomous development which integrates the public, private and academic sectors in order to ensure the use of the resources in a sustainable and competitive manner taking into account agricultural practices, water resources, technological park, non-traditional activities (tourism and aquaculture ) and government public policies.

Keywords: Viru, Partnership, Export Consortia, Theory of Commons.

\section{INTRODUCCIÓN}

La agricultura es una de las principales actividades estratégicas para el desarrollo del Perú. Los productos y derivados de la agricultura son destinados a satisfacer las necesidades alimentarias del ser humano y contribuir al crecimiento económico y social del país. De acuerdo con el INEI 2008(Instituto Nacional de Estadística e Informática), entre las principales características del sector se pueden mencionar las siguientes:

a. Empleo. La agricultura contribuye a la estabilidad económica mediante la generación de empleos y divisas. El aporte a la PEA (Población Económicamente Activa) a nivel nacional es del $26 \%$; y en el ámbito rural, del $65 \%$. El aporte al PBI (Producto Bruto Interno) es del $8 \%$.

b. Población. El $50 \%$ de la población rural depende directamente de la agricultura (población total del Perú: 28750770 habitantes; población rural: 7786624 habitantes según el censo del año 2007). Sin embargo, debido a la falta de tecnificación y capacitación existe baja productividad comparada con los indicadores regionales.

c. Superficie agrícola. Según el último censo agrario (1994), la superficie agrícola es de 5476977 ha (15\% de la superficie del Perú). La superficie sembrada en el año 2008 fue de 1 477900 ha, con un $75 \%$ de tierras en reposo.

d. Propiedad. Un tercio de la tierra destinada para uso agrícola está inscrita en los registros públicos. La mayoría de las unidades agrícolas tienen un área menor de cinco hectáreas, con altos costos de transacción, fragmentación y baja productividad.

e. Medio ambiente. Inadecuado manejo de los recursos y generación de problemas de "desertificación, deforestación, salinización, pérdida de las tierras agrícolas, toxicidad de la vegetación, agotamiento de las fuentes de agua, degradación de los ecosistemas y desaparición de especies" (Ministerio de Agricultura, 2011).

f. Organización. Entre los tipos de asociaciones agrícolas se encuentran las cooperativas, las comunidades campesinas, los pequeños y medianos productores, entre otros.

1 Doctor en Gestión de Empresas - Univ. Nacional Mayor de San Marcos Máster en Ing. Industrial e Ing. de Sistemas - Universidad de Lima Correo electrónico: chong.mario@gmail.com 
g. Mercado. Falta de información del mercado, desorden en los cultivos y cosechas de los productos. No existe una política estatal de promoción de la agricultura por otro lado, se padece de falta de información confiable para la toma de decisiones a largo plazo.

El informe "Agricultura para el desarrollo" afirma: "Con políticas adecuadas e inversiones que las respalden en el nivel local, nacional e internacional, en la actualidad este sector ofrece nuevas oportunidades para salir de la pobreza a cientos de millones de personas de las zonas rurales" (Banco Mundial, 2008). Sin embargo, de acuerdo con las características señaladas, la realidad presenta una agricultura poco desarrollada, con recursos inadecuados, baja productividad y un escaso acceso a los mercados de los pequeños productores.

\section{ANTECEDENTES}

El modelo de desarrollo del Valle de Virú consolida la visión integradora (modelo europeo) basada en la agricultura (modelo de agricultura para desarrollo y el desarrollo de una empresa agraria) y sus actividades conexas (modelo multifuncional) con una articulación entre el sector público, privado y académico en una organización flexible en procesos de incubación y promoción de la innovación y el emprendimiento (modelo de parques científicos y tecnológicos).

El modelo de complejo científico, ecológico y académico basado en la tecnología, ciencia y emprendimiento con enfoque social fue una propuesta para el desarrollo del modelo del valle de Virú y tiene como antecedentes las investigaciones sobre los bienes comunes de la Dra. Elinor Ostrom y los objetivos de desarrollo del milenio (PNUD, 2000) descritos en el apartado siguiente.

\section{OBJETIVOS DE LA INVESTIGACIÓN}

\section{Objetivo general}

Diseñar un modelo de gestión empresarial que impulse el desarrollo productivo de las pequeñas unidades agrícolas rurales en el Valle de Virú.

\section{Objetivos específicos}

- Relacionar la integración de las pequeñas unidades agrícolas, el desarrollo de la vocación agropecuaria, el desarrollo del recurso hídrico, el desarrollo del parque tecnológico y el desarrollo de la gestión pública.

- Relacionar el desarrollo de la competitividad, el desarrollo de la vocación agropecuaria, el desa- rrollo del recurso hídrico y el desarrollo de las actividades económicas no tradicionales de las pequeñas unidades agrícolas del Valle de Virú.

- Relacionar el desarrollo sostenible de las pequeñas unidades agrícolas del Valle de Virú y la integración de las políticas de los sectores público, privado y académico.

\section{3. ÁMBITO DE ESTUDIO}

La investigación se realizó en el valle de Virú, región La Libertad. El objetivo fue establecer las potencialidades y el aprovechamiento de los recursos de la región. La población de estudio estuvo conformada por las personas y organizaciones que participan en las actividades productivas, en especial, las pequeñas unidades agrícolas de Virú. Los instrumentos considerados para el análisis fueron cuatro: encuestas, entrevistas, cuestionarios y talleres.

Los actores en cada uno de los instrumentos fueron los agricultores, las autoridades locales y regionales, las organizaciones relacionadas con el manejo de los recursos hídricos, las empresas privadas y los actores representativos de la zona.

\section{HIPÓTESIS}

\section{Hipótesis general:}

El desarrollo y el crecimiento económico de todas las actividades productivas, en especial de las pequeñas unidades agrícolas, se alcanzarán en función al aumento de los niveles de integración, competitividad y desarrollo sostenible de los actores, transformando el escenario actual de desintegración, fragmentación e independencia.

\section{Hipótesis específicas:}

- El desarrollo de la vocación agropecuaria (DESAGRO), del recurso hídrico (DESHÍDRICO), del parque tecnológico (DESPARQUE) y de la gestión pública (DESPÚBLICO) favorecerán la integración de las pequeñas unidades agrícolas del Valle de Virú.

- El desarrollo de la competitividad de las pequeñas unidades agrícolas del Valle de Virú dependerá del desarrollo de la vocación agropecuaria (DESAGRO), del desarrollo del recurso hídrico (DESHÍDRICO) y del desarrollo de actividades económicas no tradicionales.

- El desarrollo sostenible de las pequeñas unidades agrícolas del valle de Virú dependerá de la integración de las políticas de los sectores público (PUB), privado (PRIV) y académico (ACAD). 


\section{INSTRUMENTOS DE LA INVESTIGACIÓN}

a) Observación: La observación se realizó en el primer trabajo de campo, fue no participante y permitió obtener una primera aproximación al contexto de la zona de estudio (Taylor, S.J. \& Bogdan, R, 2008). Asimismo, hizo posible desarrollar un alcance inicial de los siguientes aspectos:

- Las principales actividades socioeconómicas.

- La ocupación de los espacios de desarrollo económico, social y político.

- Las costumbres, conductas y códigos de comportamiento de la población.

b) Encuesta: Las encuestas de opinión son consideradas como un instrumento importante en las investigaciones no experimentales trasversales descriptivas (Hernández, 2010). Este instrumento se aplicó en el segundo trabajo de campo con cuestionarios enfocados a los productores agropecuarios.

La encuesta fue aplicada a 83 productores agropecuarios (64 varones y 19 mujeres) pertenecientes a las localidades de Huacapongo, El Niño Bajo, El Niño, Tomabal, Queneto, La Represa, Susanga, El Carmelo, La Gloria, Compositan, Zaraque, San Juan, La Calera, Santa Elena, La Gloria, San José, San Nicolás, Huancaquito Alto y Huancaquito Bajo.

El objetivo de las encuestas fue recopilar información cuantitativa y cualitativa de las actividades económicas (agricultura, ganadería, comercio), tenencia de la tierra, uso del agua, educación, salud, entre otros.

c) Entrevista: Las entrevistas tuvieron una guía general con el fin de recopilar información sobre la situación agropecuaria en el valle de Virú; de este modo, se pudieron identificar sus diferentes potencialidades y problemáticas. El formato de la entrevista fue libre, según la especialización del entrevistado, con una duración de 30 a 60 minutos, con referencias escritas y grabaciones digitales.

Los temas principales estuvieron centrados en las actividades económicas, manejo del recurso hídrico, actores representativos de la zona y alternativas de desarrollo. Las entrevistas se realizaron en los diversos trabajos de campo. En el segundo trabajo de campo, por ejemplo, se realizaron 41 entrevistas con las cuales se obtu- vo valiosa información de las potencialidades de desarrollo y de los problemas de Virú.

Los entrevistados fueron autoridades locales y regionales, representantes de las organizaciones relacionadas con el manejo de los recursos hídricos, representantes de las empresas privadas, agricultores y otros actores representativos de la zona.

d) Cuestionario: El cuestionario a los expertos, conocido como la técnica Delphi, es un instrumento de investigación útil para pronosticar el comportamiento o la situación futura de un fenómeno o una problemática.

El cuestionario anónimo se realizó en dos etapas con la participación de un grupo de opinantes seleccionados por sus conocimientos teóricos y prácticos.

La aplicación de la técnica Delphi se realizó de manera virtual con la creación de una página web, en la cual cada experto ingresaba sus respuestas en línea. Adicionalmente, se ingresó la información con la presencia de los expertos, en especial en el campo, con una conexión remota a Internet.

La primera aplicación tuvo el objetivo de validar los resultados de los instrumentos previos (observación, encuestas, entrevistas) y de validar los resultados del árbol de problemas. La segunda buscó consolidar y establecer los lineamientos de desarrollo, así como llegar al consenso con los expertos y validar los resultados de la investigación.

\section{TRABAJOS DE CAMPO}

Los trabajos de campo se realizaron en el Valle de Virú y en la ciudad de Trujillo durante un año, a partir de marzo de 2010.

Los primeros trabajos de campo fueron exploratorios, con miras a establecer la problemática, las alternativas preliminares de solución, la formulación de las hipótesis y la recopilación de la información. La Figura 1 muestra las imágenes del primer trabajo de campo en el sector urbano y rural de Virú.

El análisis situacional se realizó con el procesamiento de la información cuantitativa y cualitativa obtenida en el campo y a través de las fuentes secundarias, como los censos poblacionales y otras de corte estadístico. 
Figura 1 - Primer trabajo de campo

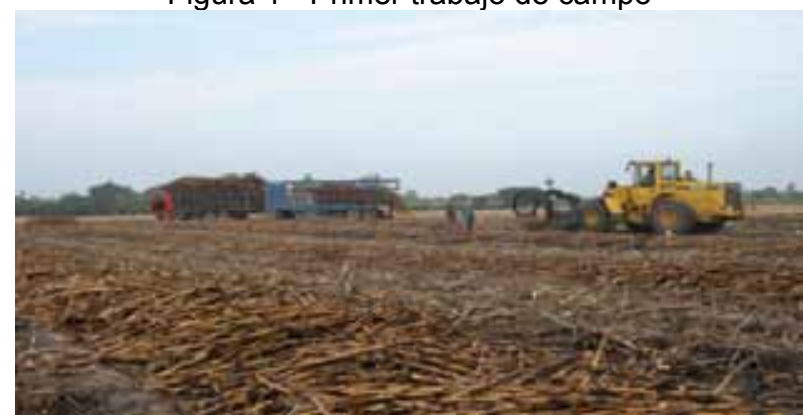

El segundo trabajo de campo tuvo como objetivo la aplicación de las encuestas y las entrevistas para la elaboración de la primera versión del árbol de problemas y el árbol de objetivos.

El análisis de la información determinó el desarrollo de la agricultura, actividad tradicional de Virú y permitió promover actividades no tradicionales como el turismo, la acuicultura, la ganadería, el manejo adecuado del recurso hídrico y la mejora de la gestión pública.

La Figura 2 muestra un espacio de desarrollo no tradicional, el puerto Morín. El cuestionario, en su primera aplicación, sirvió para validar los resultados previos y obtener la opinión de los expertos.

Figura 2 - Segundo trabajo de campo

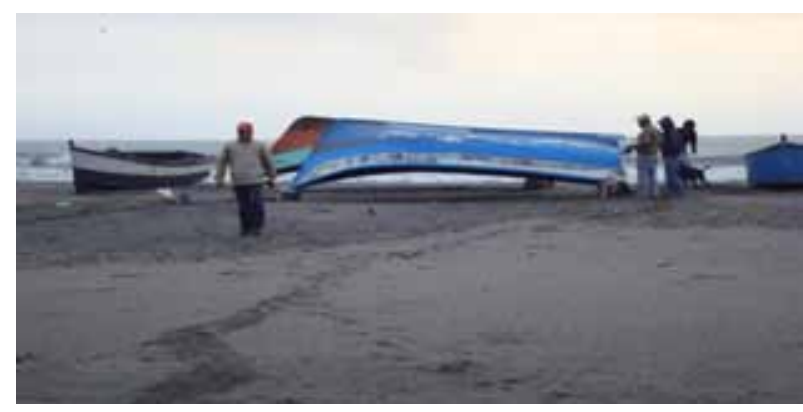

La Figura 3 muestra una imagen del tercer trabajo de campo en el proyecto especial Chavimochic y la entrevista realizada al Gerente General, el ingeniero Huber Vergara.

Figura 3 - Tercer trabajo de campo

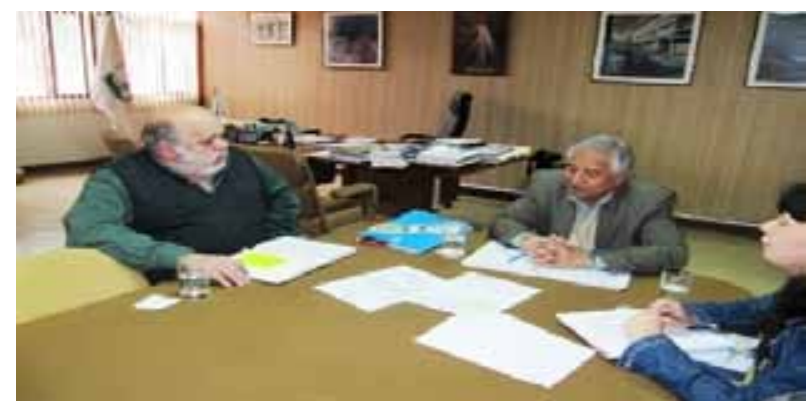

El cuarto trabajo de campo permitió concluir la primera etapa del cuestionario. Asimismo, se establecieron las pautas para la sostenibilidad de la investigación mediante la organización de talleres de asociatividad en el Valle de Virú.

Las propuestas del cuarto trabajo de campo y sus planteamientos sirvieron como base para planificar el quinto trabajo de campo y ejecutar la segunda aplicación del cuestionario. El resultado obtenido fue el informe preliminar de la investigación.

El quinto trabajo de campo, con la segunda ronda del cuestionario, permitió validar los resultados de la investigación, organizar los talleres de asociatividad y plantear el desarrollo del esquema estratégico del parque tecnológico Pro-Virú.

Figura 4 - Cuarto trabajo de campo

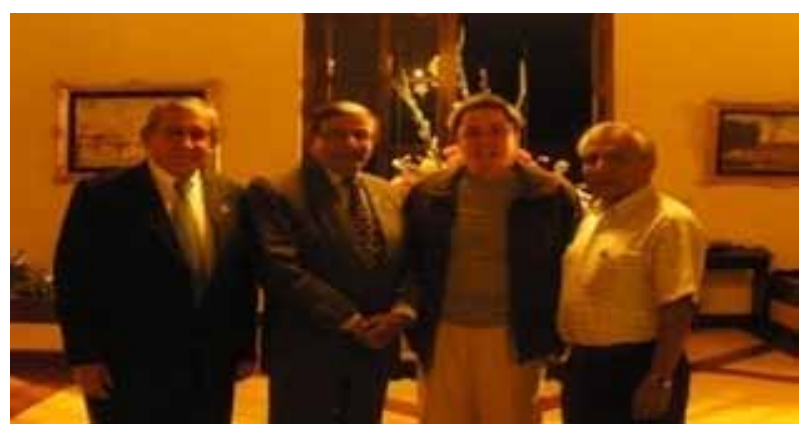

Las Figuras 5 y 6 muestran los cultivos en una zona desértica y la canalización de las aguas del río Santa.

Figura 5 - Zonas de cultivo

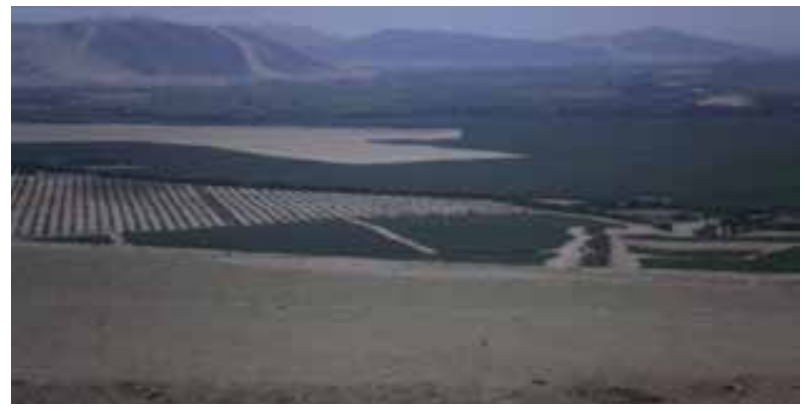

Fuente: elaboración propia (2010)

Figura 6 - Canalización del río Santa

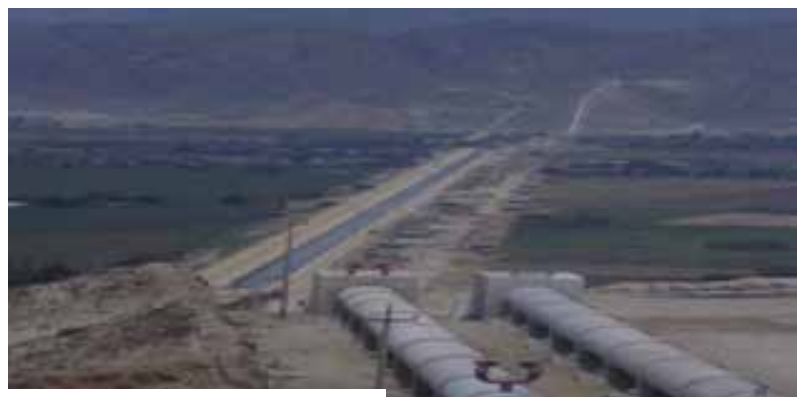




\section{TALLER DE ASOCIATIVIDAD PARA CAPACI- TADORES}

El taller de Asociatividad para capacitadores se realizó los día 25 y 26 de marzo de 2011 en coordinación con el proyecto especial Chavimochic, la unidad de posgrado de Ingeniería Industrial de la UNMSM y la Organización de las Naciones Unidas para el desarrollo (Figura 7). Fue la primera experiencia de desarrollo con la aplicación del modelo. Los objetivos propuestos fueron los siguientes:

- Establecer la sostenibilidad a la investigación mediante la capacitación a los pequeños agricultores líderes en temas de asociatividad e innovación.

- Promover la discusión y elaboración de propuestas de innovación en las pequeñas unidades agrícolas rurales.

- Consolidar la relación entre el Estado, la universidad y la empresa para el desarrollo de las pequeñas unidades agrícolas rurales del Valle de Virú.

Figura 7 - Presentación del taller

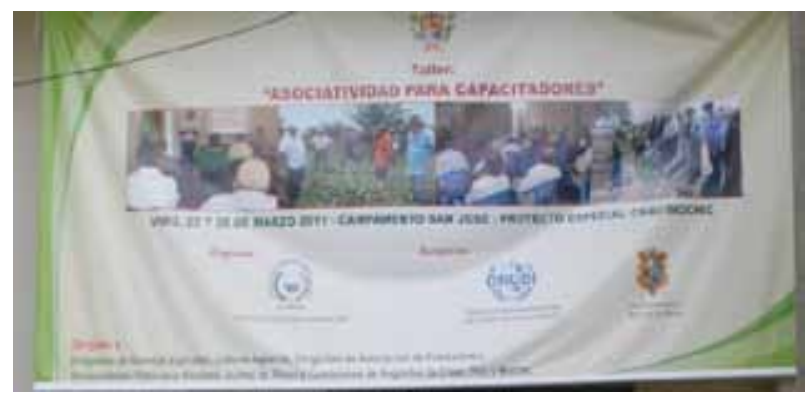

Fuente: elaboración propia (2011)

El primer día del evento estuvo dedicado a la elaboración de talleres participativos en los cuales se formaron mesas de discusión conformadas por agricultores, directivos, administradores y trabajadores de proyectos relacionados a la actividad agrícola (Figura 8).

Las discusiones siguieron tres temáticas:

1. Desafíos del entorno actual y sus alternativas. ¿Cómo es el contexto para los negocios?

2. Trabajo en redes empresariales y planes de negocio. ¿Qué podemos hacer para su desarrollo?

3. Primer esquema de soluciones.

Las ponencias se realizaron en la segunda parte del taller con la presentación y validación de la investigación en el Valle de Virú. Las ponencias fueron las siguientes:
1. Redes empresariales y financiamientos por Alejandro Siles.

2. Desarrollo y logros del proyecto especial Chavimochic por Hurber Vergara.

3. Experiencias exitosas de asociatividad por Gerardo Alegría.

4. Tecnificación de riego por Edgar Odar.

Figura 8 - Trabajo en grupos

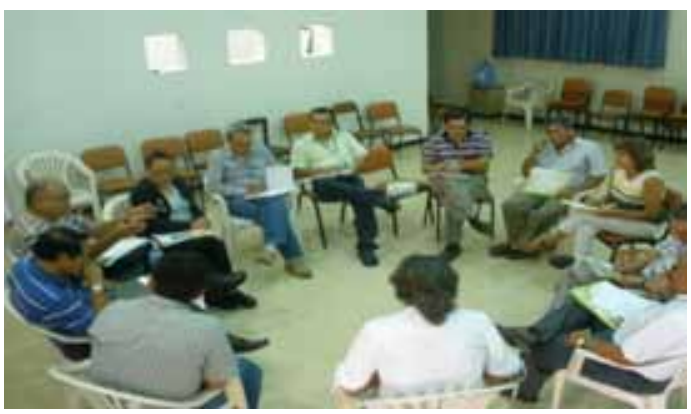

Fuente: elaboración propia (2011)

El 26 de marzo se realizaron visitas a las instalaciones del proyecto especial Chavimochic. La figura 9 muestra su aporte en el desarrollo del recurso hídrico y la Figura 10 muestra el desarrollo agroindustrial de los últimos años.

Figura 9 - Generación y transporte de agua potable

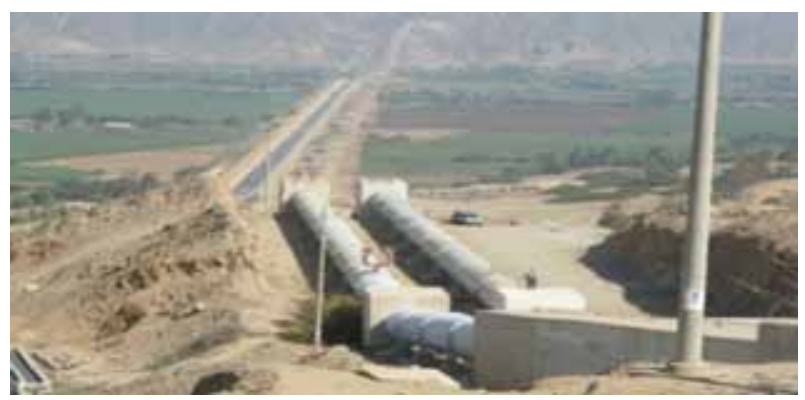

Fuente: elaboración propia (2011)

Figura 10 - Tierras ganadas al desierto

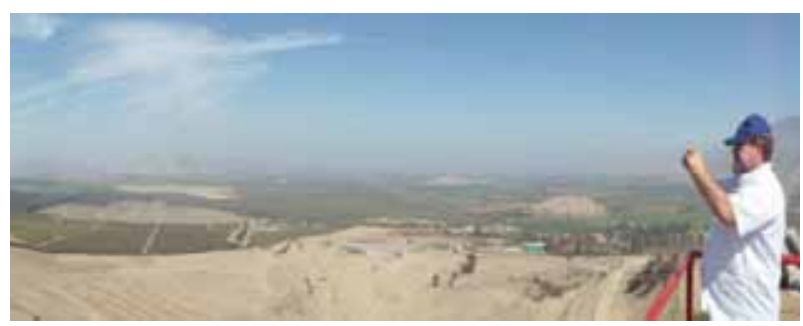

Fuente: elaboración propia (2011)

La primera visita guiada del taller se realizó a la señora Catalina Rodríguez, con una parcela de cinco hectáreas de palta fuerte en el Valle de Chao.

Según la señora Rodríguez, la experiencia de asociatividad significó el "aumento de sus ingresos por la demanda en el exterior, alcanzó poder de nego- 
ciación en el precio y la participación en una marca colectiva y logró la sostenibilidad del sector agrícola" (Figura 11).

Figura 11-Visita a la señora Catalina Rodríguez

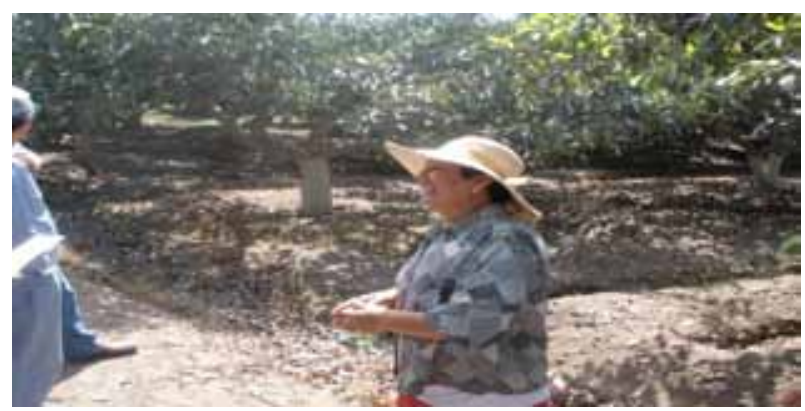

La segunda visita fue al señor Luis Sánchez Flores, quien conduce una parcela de palta orgánica en Chao. Para Sánchez "la unión es necesaria para avanzar sin problemas, salir de la crisis y afrontar con éxito la competencia" (Figura 12).

Figura 12 - Visita al señor Luis Sánchez

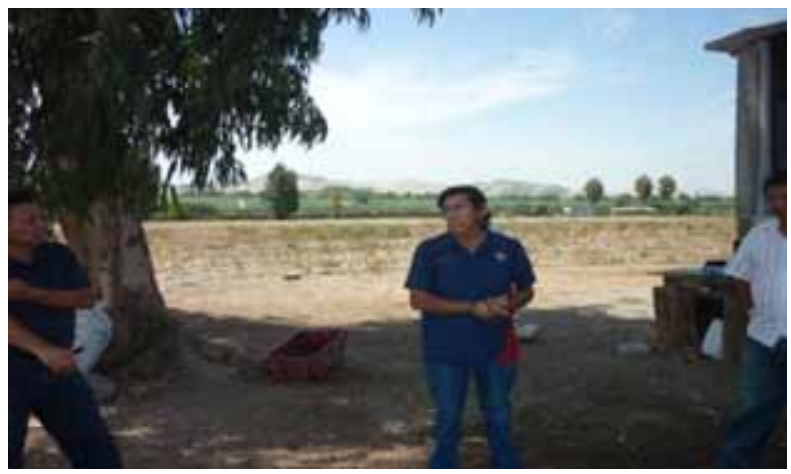

Los acuerdos del evento fueron los siguientes:

- Desarrollar proyectos de innovación tecnológica en la producción de palta, espárrago verde, mango, uva, maíz y carambola.

- Formar de un consorcio de exportación Pro-Virú.

- Regular y promocionar las marcas colectivas y consorcios de calidad.

- Continuar con la capacitación en asociatividad bajo la metodología ONUDI.

\section{TALLER DE ASOCIATIVIDAD PARA EL DESA- RROLLO RURAL INCLUSIVO}

El proyecto especial Olmos Tinajones solicitó replicar la investigación realizada en Virú y consolidar la asociatividad en la comunidad campesina de Olmos con el apoyo de la ONUDI y la Universidad Nacional Mayor de San Marcos (Figura 13).
El producto fue el taller de asociatividad para el desarrollo rural inclusivo, realizado los días 3 y 4 de noviembre de 2011. El taller contó con el auspicio del Proyecto especial Olmos Tinajones (PEOT), la Escuela de Posgrado de la UNMSM, la sociedad doctoral de Innovación y Gestión Empresarial y la ONUDI (programa de consorcios de origen en el Perú).

Los objetivos del taller fueron tres:

- Fomentar la asociatividad entre los dirigentes de gremios agrícolas, líderes y miembros de comités de gestión del valle de Olmos y los actores de la cadena productiva.

- Promover y aprovechar las oportunidades de desarrollo con la ejecución del proyecto de irrigación de Olmos-Tinajones.

- Replicar las experiencias obtenidas en el taller de asociatividad para capacitadores y proponer el modelo de desarrollo para el Valle de Olmos.

Figura 13 - Presentación del taller

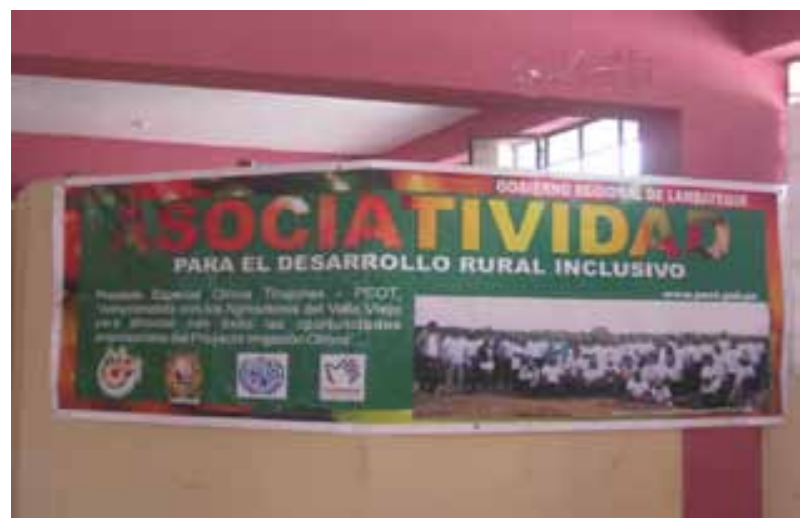

El día 3 de noviembre se realizaron reuniones con los representantes de las instituciones auspiciadoras, visitas a las instalaciones del Proyecto especial Olmos Tinajones y al Fundo experimental Pasabar con dirigentes del Valle Viejo de Olmos (Figura 14).

El día 4 de noviembre se realizaron los talleres participativos en los ambientes del Instituto Tecnológico de Olmos con la participación de más de 100 agricultores del Valle Viejo de Olmos (5 500 hectáreas), que representaban a las siete asociaciones inscritas en los Registros Públicos:

- Asociación San Isidro Labrador, Imperial.

- Asociación agropecuaria El Muerto.

- Asociación Apunkay, Tunape.

- Agropecuaria San Lorenzo, la mina Pumperre.

- Asociación Los Triunfadores, Orchilla. 
- Asociación agropecuaria Miraflores.

- Asociación agropecuaria Nitape.

Figura 14 - Visitas a las zonas de cultivo

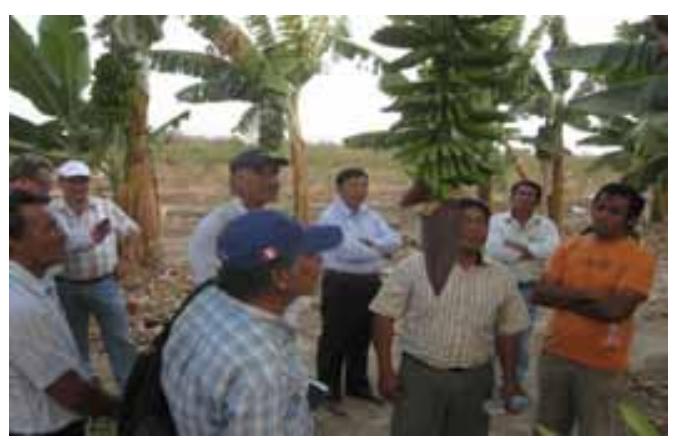

Estos talleres replicaron las experiencias del taller de asociatividad de Virú con la metodología participativa de la ONUDI (Figura 15). Las conclusiones del taller fueron las siguientes:

- Fortalezas: calidad y tenencia de la tierra.

- Oportunidad: perforación del túnel trasandino para derivar las aguas del río Huancabamba.

- Debilidad: desconfianza entre los agricultores y temor a la asociatividad.

- Amenaza: proyectos agrícolas competitivos de las empresas agroexportadoras.

Figura 15 - Taller participativo

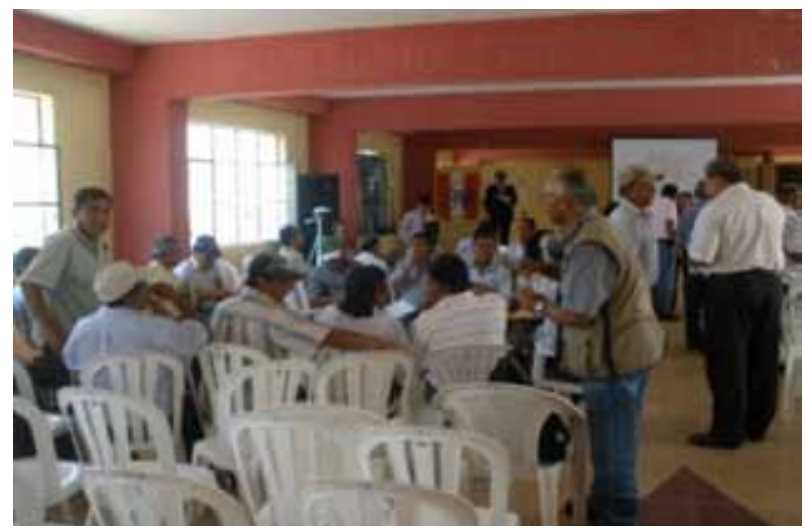

Las conclusiones de los talleres concuerdan con el aprovechamiento de las potencialidades de los valles, en función de las variables de segundo orden (DESAGRO, DESHÍDRICO, DESPARQUE, DESNOTRAD y DESPÚBLICO).

Los talleres, Figura 16, generaron espacios de diálogo entre los actores (sector público, privado y académico) para lograr el desarrollo sostenible a partir del consenso y la construcción de un entorno basado en la equidad y la asociatividad.
Figura 16 - Charla final

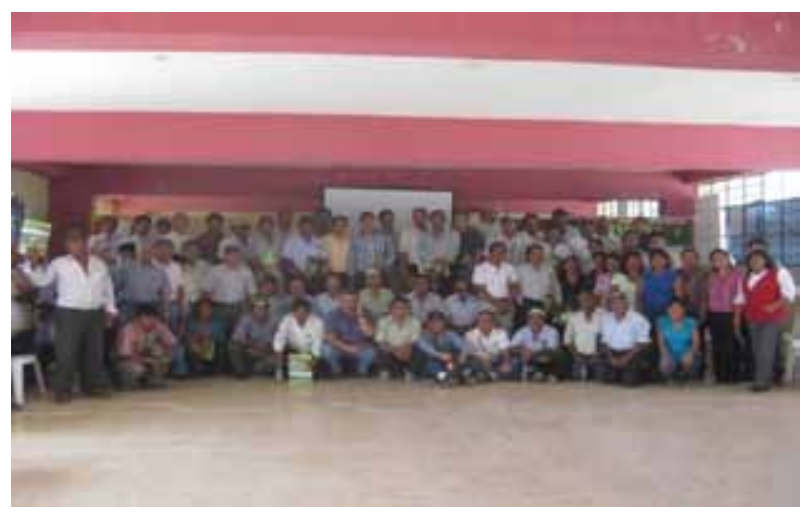

\section{CONCLUSIONES}

El estudio de los antecedentes, la metodología, los instrumentos de investigación y los talleres de asociatividad en la zona de influencia del proyecto y su réplica parcial en Olmos han demostrado las hipótesis (general y específica) del proyecto con un enfoque mixto (cuantitativo y cualitativo) por el carácter de la investigación.

La investigación confirmó la relación entre las variables, el modelo de desarrollo y el modelo operativo (Figura 17). El modelo de desarrollo de la investigación se consolidó con la organización, la ejecución y a través de los logros de los talleres de asociatividad.

Figura 17 - Modelo de desarrollo de las pequeñas unidades agrícolas (DESPUA)

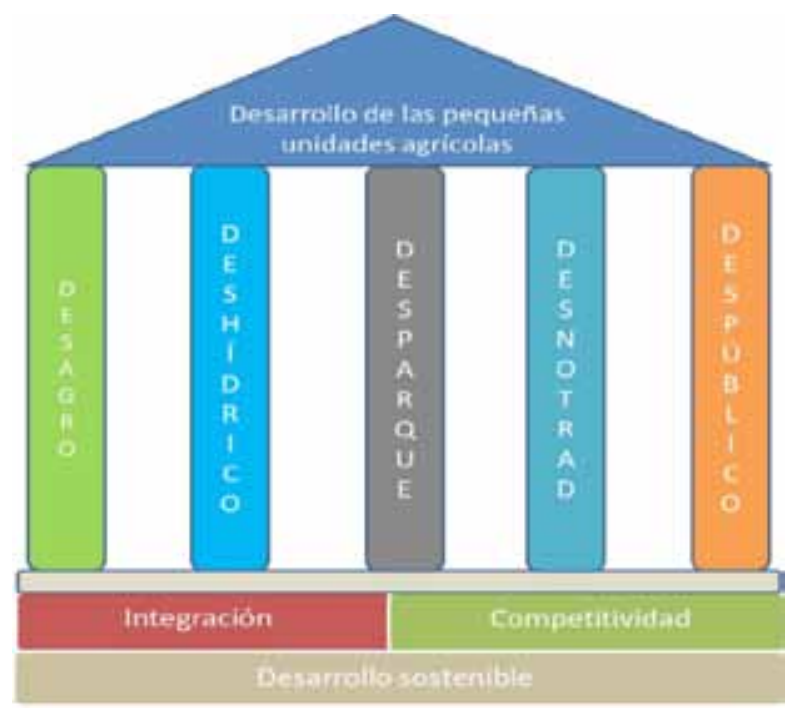




\section{REFERENCIAS BIBLIOGRÁFICAS}

[1] Banco Mundial. (2008). Agricultura para el desarrollo. Washington, DC.: Banco Mundial.

[2] Hernández-Sampieri, Fernández-Collado, \& Baptista. (2010). Metodología de la investigación. $5^{\circ}$ Edición. México DF: McGraw Hill.

[3] Instituto Nacional de Estadística e Informática. (2008). Censos Nacionales 2007: XI de población y VI de vivienda - La Libertad. Lima: INEI.

[4] Instituto Nacional de Estadística e Informática. INEI(2008). Perfil del productor agropecuario. Lima: INEI.
[5] Ministerio de Agricultura. (2011). Ministerio de Agricultura. Recuperado el 8 de 8 de 2011, de Ministerio de Agricultura: www.minag.gob.pe

[6] Programa de las Naciones Unidas para el Desarrollo. (2000). Objetivos para el desarrollo del Milenio. Recuperado el 8 de 8 de 2011. www. un.org

[7] Taylor, S. \& Bogdan, R. (1987). Introducción a los métodos cualitativos de investigación: la búsqueda de significados. Barcelona: Paidós. 